\title{
Composição corporal e exigências nutricionais de macrominerais de bovinos Caracu selecionados e Nelore selecionados ou não para peso ao sobreano ${ }^{1}$
}

\author{
Eloísio Nunes Miranda ${ }^{2}$, Augusto César de Queiroz ${ }^{3}$, Rogério de Paula Lana ${ }^{3}$, Renius Mello 4 , \\ Antônio Gesualdi Júnior ${ }^{5}$, Flávio Dutra de Resende ${ }^{6}$, Guilherme Fernando Alleoni ${ }^{6}$ \\ ${ }^{1}$ Parte da dissertação apresentada pelo primeiro autor à UFV para obtenção do título de Magister Scientiae. Trabalho realizado no APTA- \\ SP e na UFV-MG. Projeto financiado pela FAPESP. \\ 2 Mestre em Nutrição de Ruminantes. \\ ${ }^{3}$ Departamento de Zootecnia - UFV. \\ ${ }^{4}$ Doutorando em Nutrição de Ruminantes - UFV. \\ 5 TECNORTE - RJ. \\ ${ }^{6}$ Instituto de Zootecnia - SP.
}

RESUMO - Avaliaram-se a composição corporal e as exigências líquidas e dietéticas de macrominerais $(\mathrm{Ca}, \mathrm{P}, \mathrm{Mg}, \mathrm{Na}$ e K) de bovinos Caracu selecionados ( $\mathrm{CaS})$ e Nelore selecionados $(\mathrm{NeS})$ ou não $(\mathrm{NeC})$ para peso ao sobreano (378 dias de idade). Foram utilizados 56 tourinhos com média de 18 meses de idade. Doze animais foram abatidos após o período de adaptação para

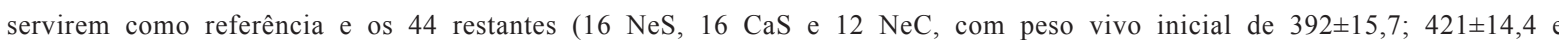
$337 \pm 18,5 \mathrm{~kg}$, respectivamente) foram distribuídos em delineamento inteiramente casualizado, em esquema fatorial $3 \times 2$ : grupo genético $(\mathrm{NeS}, \mathrm{CaS}$ e $\mathrm{NeC})$ x sistema de alimentação (restrito e ad libitum). A dieta, com relação volumoso:concentrado de 50:50, continha silagem de milho como volumoso. Os conteúdos dos macrominerais retidos no corpo dos animais foram estimados por meio de equações de regressão do logaritmo do conteúdo corporal de $\mathrm{Ca}, \mathrm{P}, \mathrm{Mg}, \mathrm{Na}$ e $\mathrm{K}$, em função do logaritmo do peso de corpo vazio (PCVZ). As exigências líquidas para ganho de $1 \mathrm{~kg}$ de PCVZ foram obtidas pela derivação das equações de predição dos conteúdos corporais dos macrominerais, em função do logaritmo do PCVZ. Houve efeito do grupo genético sobre o conteúdo corporal dos macroelementos. As concentrações corporais e as exigências de todos os macrominerais aumentaram conforme a elevação no PCVZ dos animais.

Palavras-chave: bovino de corte, cálcio, fósforo, potássio, sódio

\section{Body composition and nutritional requirements of macrominerals of genetically selected Caracu and Nellore and ordinary Nellore young bulls}

\begin{abstract}
Body composition and net and dietary requirements of macrominerals ( $\mathrm{Ca}, \mathrm{P}, \mathrm{Mg}, \mathrm{Na}$ and $\mathrm{K}$ ) of genetically selected Caracu ( $\mathrm{CaS})$ and Nellore $(\mathrm{NeS})$ for yearling weight (378 days of age) as well as of ordinary Nellore (control; $\mathrm{NeC}$ ) were investigated in this trial. Fifty-six young bulls averaging 18 months of age were used. Twelve animals were slaughtered after an adaptation period and were used as reference. The 44 remaining animals, $16 \mathrm{NeS}, 16 \mathrm{CaS}$ and $12 \mathrm{NeC}$, with initial average body weight (BW) of 392, 421 and $337 \mathrm{~kg}$, respectively, were distributed to a completely randomized design in a $3 \times 2$ factorial arrangement (genetic group [ $\mathrm{NeS}, \mathrm{CaS}$ and $\mathrm{NeC}$ ] and feeding regime [restricted and ad libitum]). Animals were fed a diet with a 50:50 forage (corn silage):concentrate ratio and were slaughtered after reach $4 \mathrm{~mm}$ of subcutaneous fat thickness measured by ultrasound. After slaughter, body parts of all animals were weighed and sampled. Samples were then freeze-dried, treated with ether to remove fat, ground and contents of macrominerals determined. Estimation of body retained macrominerals were done by regressing the logarithm of $\mathrm{Ca}, \mathrm{P}, \mathrm{Mg}, \mathrm{Na}$ and $\mathrm{K}$ body content on the logarithm of empty body weight (EBW). Net macrominerals requirements for $1 \mathrm{~kg}$ of EBW gain were obtained by derivation of the prediction equations. There was a significant effect of genetic group on the body content of the studied macrominerals. Body contents and requirements of all macrominerals followed EBW and increased as EBW of animals increased.
\end{abstract}

Key Words: beef cattle, calcium, phosphorus, potassium, sodium

\section{Introdução}

Apesar de constituírem apenas $4 \%$ do peso corporal dos animais, os minerais exercem funções vitais no organismo, com reflexos no desempenho animal. Deficiências de um ou mais elementos minerais podem resultar em desordens nutricionais sérias, levando o animal a desempenhos produtivo e reprodutivo aquém de seu potencial. 
A determinação dos requerimentos de minerais para bovinos em crescimento é extremamente complexa, em virtude dos diversos fatores que influenciam de forma direta ou indireta sua utilização pelo animal. Entre estes fatores, alguns são inerentes aos alimentos ou às dietas - disponibilidade e forma química do mineral nos ingredientes da dieta; inter-relações (antagonismos e agonismos) entre os minerais; correlações entre as frações orgânica e inorgânica; interações com outros nutrientes - e ao animal - peso corporal, variabilidade entre as raças, nível de produção e status nutricional (ARC, 1980; Underwood, 1981; Conrad et al., 1985; AFRC, 1991; Coelho da Silva, 1995; Fontes, 1995; NRC, 1996).

Além do suprimento adequado de minerais, o AFRC (1991) salienta que níveis adequados de proteína e energia são necessários para que ocorra o desenvolvimento normal dos ossos. O NRC (1996) relaciona os requerimentos de Ca e P ao ganho diário de proteína e Conrad et al. (1985) afirmam que a nutrição adequada destes minerais depende do nível de vitamina $\mathrm{D}$ da dieta.

A retenção de minerais depende da composição do ganho de peso (ossos, músculo e gordura). Maiores deposições de gordura reduzem as deposições de minerais e, conseqüentemente, seus requisitos pelos animais, já que a concentração destes elementos inorgânicos no tecido adiposo é menor que nos músculos e ossos. Portanto, fatores que modificam a composição do ganho, como tipo de dieta, sexo, grupo genético, idade e peso dos animais, afetam a composição mineral e, conseqüentemente, os requerimentos líquidos para ganho (Paulino et al., 1999).

As exigências nutricionais de macroelementos minerais para crescimento e engorda são, geralmente, estimadas pelo método fatorial (ARC, 1980), que se baseia nas quantidades líquidas depositadas no corpo do animal para crescimento e engorda, acrescidas das quantidades necessárias para atender às perdas inevitáveis do corpo, ou seja, às secreções endógenas, que são as exigências líquidas de mantença. A soma das frações de mantença e produção constitui a exigência líquida total, que, corrigida por um coeficiente de absorção do elemento inorgânico no aparelho digestivo do animal, resulta na exigência dietética do referido mineral (Coelho da Silva, 1995).

O ARC (1980) admite que os requerimentos líquidos de macroelementos minerais são constantes, independentemente do peso do animal, concluindo ainda que não há evidências de queda nas suas concentrações com o aumento do peso de corpo vazio ( $\mathrm{PCVZ}$ ) e estimando os requerimentos de $\mathrm{Ca}, \mathrm{P}, \mathrm{Mg}$, Na e K em 14,0; 8; 0,45; 1,5 e 2, $0 \mathrm{~g} / \mathrm{kg}$ de ganho de peso de corpo vazio (GPCVZ), respectivamente.
O AFRC (1991), por sua vez, reexaminando o modelo adotado pelo ARC (1980), propôs uma nova metodologia para determinação dos requisitos de $\mathrm{Ca} \mathrm{e} \mathrm{P}$ que introduz o peso à maturidade, $\mathrm{o} \mathrm{PV}$, em vez do $\mathrm{PCVZ}$, e o ganho de peso e adota equações baseadas no crescimento ósseo. Por meio desta metodologia, foi possível verificar decréscimo na deposição de Ca e $\mathrm{P}$ à medida que o PV se eleva, uma vez que ocorre redução acentuada na proporção de ossos na carcaça. Este conselho estimou as exigências líquidas de Ca e P para ganho de peso de bovinos em crescimento, considerando-se peso à maturidade de $900 \mathrm{~kg} \mathrm{em}$, respectivamente, 11,3 e 6,6 g/dia, para bovinos de $500 \mathrm{~kg}$ de PV, ganhando $1 \mathrm{~kg}$ de $\mathrm{PV} /$ dia.

O NRC (1996) estimou as exigências líquidas de Ca e P para ganho de peso em função da retenção diária de proteína, estabelecendo uma razão de 7,1 g de Ca e de 3,9 g de P para cada $100 \mathrm{~g}$ de proteína retida no corpo animal, sendo que, para animais com $450 \mathrm{~kg}$ de $\mathrm{PV}$, as exigências líquidas de $\mathrm{Ca}$ e P foram estimadas em 8,5 e 4,8 g/dia, respectivamente, para ganho de $1 \mathrm{~kg}$ de PV por dia. Este conselho calculou as exigências líquidas de $\mathrm{Ca}$ e $\mathrm{P}$ para mantença em função do $\mathrm{PV}$ do animal, as quais seriam, respectivamente, de 15,4 e $16 \mathrm{mg} / \mathrm{kg}$ de PV/dia.

De acordo com esse sistema, as exigências de $\mathrm{Mg}$ para mantença foram calculadas em $3 \mathrm{mg} / \mathrm{kg}$ de $\mathrm{PV} /$ dia e a exigência dietética para animais em crescimento e terminação ganhando $1 \mathrm{~kg}$ de PV/dia, de $0,10 \%$ do elemento na MS total da dieta, considerando consumo de 2,4\% do PV de MS. Para Na e K, as exigências dietéticas recomendadas são, respectivamente, de 0,06 a 0,08 e $0,6 \%$ na MS da dieta.

Para converter as exigências líquidas em exigências dietéticas, é preciso conhecer o coeficiente de absorção ou a biodisponibilidade do mineral considerado, o qual estima o quanto do elemento inorgânico consumido será absorvido pelo animal. O AFRC (1991) adotou valores de disponibilidade de $68 \%$ para $\mathrm{Ca}$, enquanto, para $\mathrm{P}$, relacionou a disponibilidade com a metabolizabilidade (q) da dieta ( $q=$ energia bruta/energia metabolizável), adotando valores de $58 \%$, para $q<0,70$ e de $70 \%$, para $q>0,70$. O NRC (1996) recomenda valores médios para Ca e $\mathrm{P}$ de 50 e $68 \%$, respectivamente, e uma faixa de absorção para $\mathrm{Mg}$ de 10 a 37\%, não apresentando valores para $\mathrm{Na}$ e K. O ARC (1980) relata coeficientes de absorção de 91 e 100\% para Na e K, respectivamente.

As informações sobre a composição corporal e, conseqüentemente, sobre os requerimentos de macroelementos minerais para bovinos de corte de diferentes grupos genéticos, em especial zebuínos e Caracu selecionados em situações brasileiras, ainda são escassas. Dessa forma, objetivou-se 
com este estudo avaliar a composição corporal e as exigências líquidas e dietéticas de macroelementos minerais $(\mathrm{Ca}, \mathrm{P}, \mathrm{Mg}$, $\mathrm{Na}$ e K) de bovinos Nelore e Caracu selecionados para peso aos 378 dias de idade e Nelore não-selecionados.

\section{Material e Métodos}

O experimento foi realizado na Estação Experimental de Zootecnia de Colina, do Instituto de Zootecnia de São Paulo. Foram utilizados 56 novilhos não-castrados, com média de 18 meses de idade, provenientes de amostras retiradas da 19a progênie de rebanhos, sendo 20 Nelore $(\mathrm{NeS})$ e $20 \mathrm{Caracu}(\mathrm{CaS})$ submetidos à seleção com base no peso aos 378 dias de idade ao final de prova de ganho de peso. Foram utilizados ainda 16 Nelore não-selecionados $(\mathrm{NeC})$ nos quais não foi aplicado o método de seleção.

Após período de adaptação de 28 dias, em que todos os animais receberam a ração utilizada no período experimental, quatro animais de cada grupo genético foram abatidos e serviram como referência para estimativa do PCVZ e da composição corporal inicial dos animais remanescentes. Os 44 animais restantes entraram no ensaio de alimentação apresentando peso vivo (PV) inicial de $392 \pm 15,7 \mathrm{~kg}$ para $\mathrm{NeS}, 337 \pm 14,4 \mathrm{~kg}$ para $\mathrm{NeC}$ e $421 \pm 18,5 \mathrm{~kg}$ para CaS. Os animais foram distribuídos em baias individuais, de modo que metade dos animais de cada grupo genético (oito $\mathrm{NeS}$, seis $\mathrm{NeC}$ e oito $\mathrm{CaS}$ ) recebeu alimentação ad libitum até o abate e a outra metade recebeu alimentação restrita, na expectativa de se fixar o consumo em $65 \mathrm{~g} \mathrm{MS} / \mathrm{kgPV}^{0,75}$.

A alimentação era fornecida duas vezes ao dia, efetuando-se o controle diário do consumo de alimentos, permitindo-se quantidade máxima de sobra em torno de $5 \%$ do oferecido (ad libitum) para os animais. A ração fornecida foi balanceada com silagem de milho, milho moído, farelo de algodão, uréia, rumensin e mistura mineral, em uma relação volumoso/concentrado, de 50/50 na MS. Os teores de nutrientes digestíveis totais e de EM foram de 79\% na MS e $2,85 \mathrm{Mcal} / \mathrm{kg} \mathrm{MS}$, respectivamente. A dieta foi reajustada semanalmente com base no consumo de MS e na porcentagem de MS do volumoso e do concentrado. Os níveis de nutrientes dos componentes da ração, determinados conforme metodologias descritas por Silva \& Queiroz (2002), são apresentados na Tabela 1.

Os animais foram abatidos quando atingiram, no mínimo, $4 \mathrm{~mm}$ de espessura de gordura subcutânea (avaliada por ultra-som) sobre o músculo Longissimus dorsi entre a 12 a e 13 - costelas. Para o peso final no dia do abate, foi realizado jejum completo de 18 horas. Após o abate, o trato gastrintestinal de cada animal foi esvaziado, lavado e seu peso foi somado ao dos demais componentes do corpo (carcaça, cabeça, couro, pés, cauda, sangue, vísceras e órgãos), determinando-se o PCVZ. As relações específicas entre o PCVZ e o PV dos animais-referência de cada grupo genético foram utilizadas para estimativa do PCVZ inicial dos animais remanescentes.

Os tecidos corporais foram acondicionados em sacos plásticos e congelados, à exceção da amostra de couro, que foi resfriada em câmara fria. A carcaça de cada animal foi dividida em duas meia-carcaças, as quais foram pesadas individualmente e resfriadas a $-5^{\circ} \mathrm{C}$ em câmara fria por 18 horas, sendo pesadas novamente após este período. Para determinação da porcentagem dos tecidos da carcaça, utilizou-se a metodologia descrita por Hankins \& Howe (1946).

Após congelamento ou resfriamento, os componentes corporais foram reduzidos a pedaços menores, posteriormente moídos e homogeneizados, retirando-se, então,

Tabela 1- Teores de MS, MO, PB, FDN, FDNcp, CHOT, CNF, EE, Ca, P, Mg, Na e K da mistura do concentrado e dos ingredientes da dieta Table 1 - Contents of DM, OM, CP, NDF, NDFcp, TCHO, NFC, EE, Ca, P, Mg, Na and K of the concentrate mixture and ingredients of the diet

\begin{tabular}{|c|c|c|c|c|}
\hline $\begin{array}{l}\text { Nutriente } \\
\text { Nutrient }\end{array}$ & $\begin{array}{l}\text { Mistura concentrada } \\
\text { Concentrate mixture }\end{array}$ & $\begin{array}{l}\text { Farelo de algodão } \\
\text { Cottonseed meal }\end{array}$ & $\begin{array}{l}\text { Milho moído } \\
\text { Ground corn }\end{array}$ & $\begin{array}{c}\text { Silagem de milho } \\
\text { Corn silage }\end{array}$ \\
\hline $\operatorname{MS}(D M)(\%)^{1}$ & 81,70 & 81,94 & 79,57 & 27,14 \\
\hline $\mathrm{PB}(C P)^{1,2}$ & 18,81 & 34,40 & 9,46 & 8,82 \\
\hline FDN $(N D F)^{1,2}$ & 15,98 & 36,96 & 12,56 & 60,92 \\
\hline FDNcp $(\text { NDFap })^{1,2}$ & 13,97 & 33,98 & 11,06 & 57,69 \\
\hline CHOT $(\text { TCHO })^{1}$ & 72,67 & 55,92 & 86,06 & 84,88 \\
\hline $\mathrm{EE}^{1,2}$ & 3,81 & 4,18 & 3,03 & 2,25 \\
\hline $\mathrm{Ca}^{2}$ & 0,56 & 0,48 & 0,02 & 0,65 \\
\hline $\mathrm{P}^{2}$ & 0,29 & 0,99 & 0,14 & 0,18 \\
\hline $\mathrm{Mg}^{2}$ & 0,23 & 0,64 & 0,10 & 0,54 \\
\hline $\mathrm{Na}^{2}$ & 0,24 & 0,04 & 0,01 & 0,01 \\
\hline $\mathrm{K}^{2}$ & 0,40 & 0,48 & 0,26 & 1,81 \\
\hline
\end{tabular}

1 Dados de Gesualdi Jr. (2003) (Data from Gesualdi Jr. [2003]).

$2 \%$ na MS (\% of DM). 
amostras de cada componente de cada animal. As amostras dos componentes e do sangue foram levadas ao liofilizador, por aproximadamente 80 horas, para determinação da matéria seca gordurosa (MSG).

As amostras de carcaça, couro, vísceras, cabeça e pés foram submetidas à extração de gordura com éter de petróleo, obtendo-se a matéria seca pré-desengordurada (MSPD). Em seguida, este material foi novamente triturado em moinho de bola e acondicionado em potes plásticos. A amostra de sangue não sofreu pré-desengorduramento e foi moída em liquidificador com gelo seco.

A solução mineral para determinação dos macroelementos minerais nos tecidos constituintes do corpo vazio dos animais foi preparada pela técnica por via úmida, de acordo com metodologia descrita por Silva \& Queiroz (2002). Os conteúdos corporais dos macroelementos minerais foram determinados na MSPD considerando-se suas concentrações percentuais nos tecidos de constituição do corpo vazio dos animais (carcaça, vísceras, órgãos, couro, sangue, cabeça, pés e cauda).

Para predição das quantidades líquidas dos macroelementos minerais retidos no corpo vazio dos animais de cada tratamento (grupo genético) e para os tratamentos em conjunto, foram ajustadas equações de regressão do logaritmo do conteúdo corporal de $\mathrm{Ca}, \mathrm{P}, \mathrm{Mg}$, $\mathrm{Na}$ e $\mathrm{K}$, em função do logaritmo do PCVZ, segundo o ARC (1980), conforme o seguinte modelo:

$$
\mathrm{Y}_{\mathrm{ij}}=\mu+\mathrm{b}_{\mathrm{i}} \mathrm{X}_{\mathrm{ij}}+\mathrm{e}_{\mathrm{ij}} \text {, }
$$

em que: $Y_{i j}=$ logaritmo do conteúdo total do macroelemento mineral (kg) retido no corpo vazio do animal $\mathrm{j}$ do grupo genético $i ; \mu=$ efeito da média (intercepto); $b_{i}=$ coeficiente de regressão do logaritmo do conteúdo corporal do macroelemento mineral, em função do logaritmo do PCVZ, para o grupo genético i; em que $\mathrm{i}=1$, Nelore selecionado; 2, Nelore não-selecionado; 3, Caracu selecionado; $\mathrm{X}_{\mathrm{ij}}=$ logaritmo do PCVZ, do animal j do grupo genético i; $\mathrm{e}_{\mathrm{ij}}=$ erro aleatório associado à observação, suposto normal e independentemente distribuído com média $=0$ e variância $=\sigma^{2}$, ou seja, $\mathrm{e}_{\mathrm{ij}} \sim N\left(0 ; \sigma^{2}\right)$.

Em cada tratamento, as equações foram obtidas adicionando-se os valores relativos aos animais-referência.

Derivando-se as equações de predição dos conteúdos corporais dos macroelementos minerais, em função do logaritmo do PCVZ, foram obtidas as equações de predição das exigências líquidas de $\mathrm{Ca}, \mathrm{P}, \mathrm{Mg}$, $\mathrm{Na}$ e K para ganho de $1 \mathrm{~kg}$ de PCVZ, segundo o ARC (1980), conforme o modelo:

$$
\mathrm{Y}^{\prime}=\mathrm{b} \cdot 10^{\mathrm{a}} \cdot \mathrm{X}^{(\mathrm{b}-1)}
$$

em que: $\mathrm{Y}^{\prime}=$ exigência líquida do macroelemento mineral $(\mathrm{g})$; $\mathrm{a}$ e $\mathrm{b}=$ intercepto e coeficiente de regressão, respectivamente, das equações de predição dos conteúdos corporais dos macroelementos minerais; $\mathrm{X}=\mathrm{PCVZ}(\mathrm{kg})$.

Para se estimarem as exigências líquidas de mantença de cada macroelemento mineral e, posteriormente, somá-las às exigências líquidas para ganho, obtendo-se as exigências dietéticas totais, foram adotadas as recomendações para as perdas endógenas totais de $\mathrm{Ca}, \mathrm{P}, \mathrm{Mg}, \mathrm{Na}$ e $\mathrm{K}$ e as biodisponibilidades destes elementos nos alimentos segundo o ARC (1980) e o NRC (1996), conforme Tabela 2.

A conversão do PV em PCVZ, dentro do intervalo de pesos incluídos no trabalho, foi feita utilizando-se a equação obtida pela regressão do PCVZ dos 56 animais utilizados no experimento, em função do PV. Para conversão das exigências líquidas para ganho de PCVZ em exigências líquidas para ganho, utilizou-se o fator obtido a partir dos dados experimentais.

Os resultados foram interpretados estatisticamente por meio de análises de variância e regressão, utilizando-se o Sistema de Análises Estatísticas e Genéticas - SAEG (UFV, 1998), aplicando-se o teste $F$ a $5 \%$ de probabilidade na análise de variância. As comparações entre as equações de regressão dos parâmetros avaliados para cada grupo genético foram realizadas de acordo com a metodologia proposta por Regazzi (1996), para testar a identidade de modelos.

Tabela 2 - Perdas endógenas totais e biodisponibilidade média de $\mathrm{Ca}, \mathrm{P}, \mathrm{Mg}, \mathrm{Na}$ e $\mathrm{K}$ nos alimentos

\begin{tabular}{|c|c|c|}
\hline $\begin{array}{l}\text { Elemento mineral } \\
\text { Mineral element }\end{array}$ & $\begin{array}{l}\text { Perda endógena total } \\
\text { Total endogenous losses }\end{array}$ & $\begin{array}{c}\text { Biodisponibilidade }(\%) \\
\text { Bioavailability }\end{array}$ \\
\hline $\begin{array}{l}\mathrm{Ca} \\
\mathrm{P} \\
\mathrm{Mg} \\
\mathrm{Na} \\
\mathrm{K}\end{array}$ & $\begin{array}{c}15,4 \mathrm{mg} / \mathrm{kg} \mathrm{PV} / \text { dia }(B W / \text { day })^{1} \\
16 \mathrm{mg} / \mathrm{kg} \mathrm{PV} / \text { dia }(B W / \text { day })^{1} \\
3,0 \mathrm{mg} / \mathrm{kg} \mathrm{PV} / \text { dia }(B W / \text { day })^{2} \\
6,8 \mathrm{mg} / \mathrm{kg} \mathrm{PV} / \text { dia }(B W / \text { day })^{2} \\
\text { Fecal }-2,6 \mathrm{~g} / \mathrm{kg} \mathrm{MS} \mathrm{consumida}(\text { DM intake })^{2,3} \\
\text { Urinária (Urinary) }-37,5 \mathrm{mg} / \mathrm{kg} \mathrm{PV/dia}(B W / \text { day })^{2} \\
\text { Salivar (Salivary) }-0,7 \mathrm{~g} / 100 \mathrm{~kg} \mathrm{PV/dia}(\text { BW/day })^{2} \\
\text { Através da pele (From skin }-1,1 \mathrm{~g} / \text { dia }(\text { g/day })^{2}\end{array}$ & $\begin{array}{l}50^{1} \\
68^{1} \\
17^{2} \\
91^{2} \\
100^{2}\end{array}$ \\
\hline
\end{tabular}

Table 2 - Total endogenous losses and average bioavailability of $\mathrm{Ca}, \mathrm{P}, \mathrm{Mg}, \mathrm{Na}$, and $\mathrm{K}$ in the feeds

\footnotetext{
1 Dados obtidos do NRC (1996) (Data from NRC, 1996).

2 Dados obtidos do ARC (1980) (Data from ARC, 1980).

${ }^{3}$ Considerando consumo de MS de $2,4 \%$ do PV (Assuming DM intake of $2.4 \%$ BW).
} 


\section{Resultados e Discussão}

A equação geral obtida para predição do peso de corpo vazio (PCVZ), em função do peso vivo (PV), ajustada para os três grupos genéticos em conjunto, foi: $\mathrm{PCVZ}=-32,65$ $+0,9668 . \mathrm{PV} ; \mathrm{r}^{2}=0,98$. O elevado valor do coeficiente de determinação demonstra bom ajustamento da equação aos dados. Esta equação baliza valores médios para o PCVZ em $89,6 \%$ do $\mathrm{PV}$, próximos àqueles recomendados pelo NRC (1996), de $89,1 \%$.

A partir dessa equação, foi obtida a equação para estimativa do PV, em função do PCVZ, objetivando possibilitar a conversão das exigências líquidas para ganho de PCVZ em exigências líquidas para ganho de PV. A equação obtida foi: $\mathrm{PV}=1,03$ (PCVZ + 32,65). Portanto, nas condições deste experimento, o ganho de $1 \mathrm{~kg}$ de PCVZ correspondeu ao de $1,03 \mathrm{~kg}$ de PV.

Os parâmetros das equações de regressão do logaritmo dos conteúdos de $\mathrm{Ca}, \mathrm{P}, \mathrm{Mg}$, $\mathrm{Na}$ e $\mathrm{K}$ no corpo vazio, em função do logaritmo do PCVZ, podem ser observados na Tabela 3.

O teste de identidade de modelos (Regazzi, 1996) aplicado às equações de regressão do logaritmo do conteúdo corporal dos macroelementos minerais ( $\mathrm{Ca}, \mathrm{P}, \mathrm{Mg}, \mathrm{Na}$ e K), em função do logaritmo do PCVZ, para os três grupos genéticos estudados, revelou não haver diferenças $(\mathrm{P}>0,05)$ entre as equações ajustadas para $\mathrm{P}, \mathrm{Mg}$ e Na para Nelore selecionado ( $\mathrm{NeS}$ ) e Nelore não-selecionado $(\mathrm{NeC})$, as quais diferiram das equações ajustadas para Caracu selecionado $(\mathrm{CaS})$. Desta forma, são apresentadas equações comuns para $\mathrm{P}, \mathrm{Mg}$ e Na para $\mathrm{NeS}$ e $\mathrm{NeC}$ e específicas para CaS. Para $\mathrm{Ca}$ e K, esse teste revelou não haver diferenças $(\mathrm{P}>0,05)$ entre as equações ajustadas para $\mathrm{NeC}$ e $\mathrm{CaS}$, as quais diferiram daquelas referentes ao NeS. Portanto, adotaram-se equações comuns para $\mathrm{Ca}$ e $\mathrm{K}$ para $\mathrm{NeC}$ e $\mathrm{CaS}$ e específicas para NeS.

Os coeficientes de determinação $\left(\mathrm{r}^{2}\right)$ das equações de regressão, de modo geral, foram altos, indicando ajuste elevado dos modelos para estimar a composição dos animais em estudo. Os conteúdos corporais de $\mathrm{Ca}, \mathrm{P}, \mathrm{Mg}, \mathrm{Na}$ e K por unidade de peso corporal vazio ( $\mathrm{g} / \mathrm{kg}$ de $\mathrm{PCVZ}$ ) são apresentados na Tabela 4.

Observou-se maior conteúdo corporal para todos os minerais estudados para animais $\mathrm{NeS}$ em relação aos animais $\mathrm{CaS}$. Estes resultados não eram esperados, uma vez que Gesualdi Jr. et al. (2006), analisando a composição física da carcaça dos animais utilizados neste trabalho, observaram maior proporção de tecido muscular e menor de tecido adiposo para animais $\mathrm{CaS}$ em relação aos animais $\mathrm{NeS}$, os quais não diferiram dos $\mathrm{NeC}$. Quanto ao tecido ósseo, esse autor não observou diferenças significativas entre os três grupos genéticos. Assim, esperava-se menor conteúdo corporal de minerais para os animais $\mathrm{NeS}$ e $\mathrm{NeC}$ em relação aos CaS, emvirtude dos maiores depósitos de gordura encontrados nos $\mathrm{NeS}$, os quais exercem efeito de diluição nos conteúdos de minerais, conseqüência do baixo teor de

Tabela 3 - Parâmetros das equações de regressão do logaritmo do conteúdo corporal $(\mathrm{kg})$ de Ca, P, Mg, Na e K no corpo vazio, em função do logaritmo do peso de corpo vazio, em $\mathrm{kg}$, para bovinos Nelore e Caracu ${ }^{1}$

Table 3 - Coefficients of the logarithmical regression equations of body contents ( $\mathrm{kg}$ ) of $\mathrm{Ca}, \mathrm{P}, \mathrm{Mg}, \mathrm{Na}$, and $\mathrm{K}$ on the logarithm of empty body weight, in $\mathrm{kg}$, of Nellore and Caracu

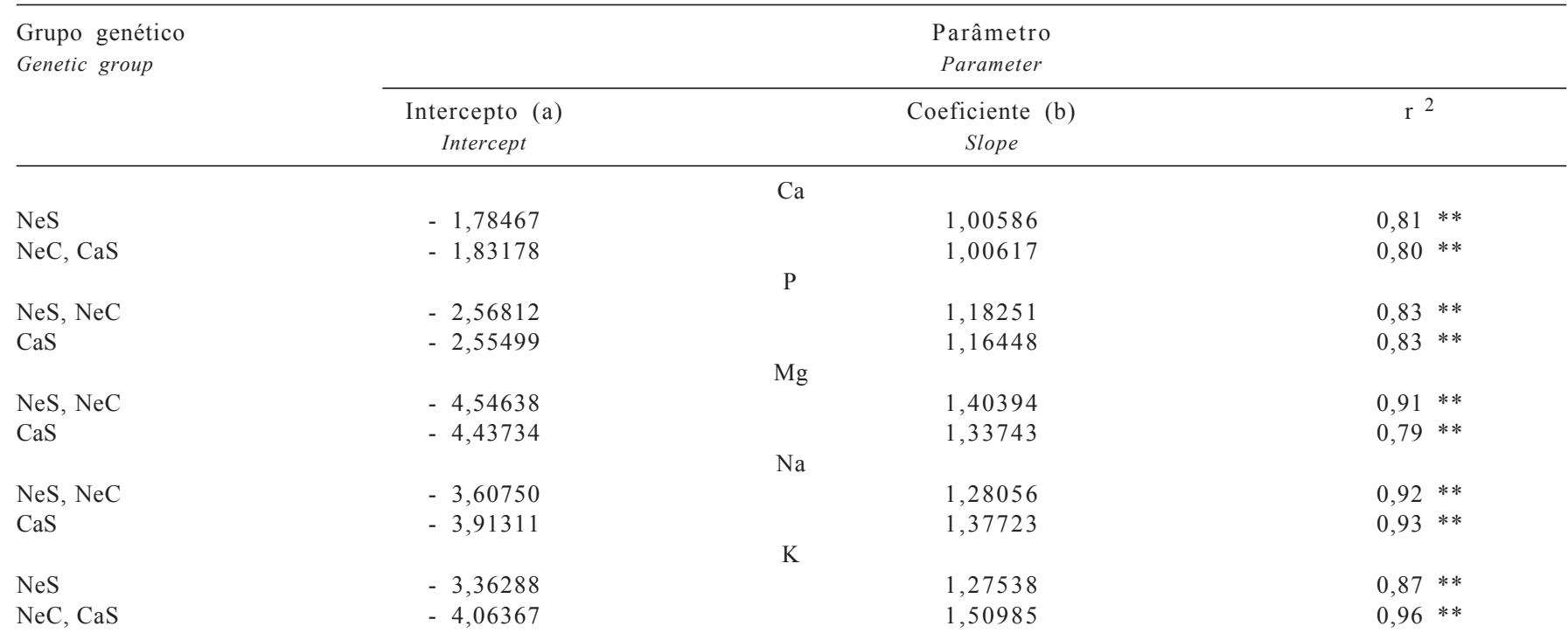

$1 \mathrm{NeS}=$ Nelore selecionado; $\mathrm{NeC}=$ Nelore não-selecionado; CaS = Caracu selecionado.

$1 \mathrm{NeS}=$ Genetically selected Nellore; NeC = Ordinary Nellore ; CaS = Genetically selected Caracu .

** $\mathrm{P}<0,01(P<.01)$ 
minerais no tecido adiposo. Os animais $\mathrm{NeS}$ e $\mathrm{NeC}$, por sua vez, não deveriam diferir entre si, visto que não houve diferenças na sua composição física da carcaça.

Quanto aos conteúdos corporais e às exigências líquidas e dietéticas, apresentadas posteriormente neste trabalho, as diferenças entre os grupos genéticos foram menos marcantes que entre as variações de peso corporal. Além disso, estas diferenças podem ser mascaradas pela falta de uniformidade dos resultados publicados na literatura.

Os conteúdos corporais dos cinco macrominerais estudados aumentaram com a elevação do peso corporal dos animais, o que diverge dos dados normalmente encontrados na literatura, que relatam decréscimo nas concentrações dos macrominerais no corpo vazio com a elevação do PCVZ, em decorrência do efeito de diluição provocado pelo aumento da gordura corporal, a qual apresenta menor concentração de minerais que os tecidos ósseo e muscular. Além disso, Fontes (1995) concluiu que há também redução acentuada na proporção de ossos na carcaça com a elevação do PCVZ, o que contribui para esta redução no teor de minerais. O comportamento observado neste trabalho difere do relatado pelo AFRC (1991), por Lana et al. (1992), Coelho da Silva (1995), Fontes (1995), Paulino et al. (1999) e Paulino et al. (2004).

A possível explicação para o aumento das concentrações dos minerais com a elevação do peso corporal dos animais pode estar relacionada ao fato de que esses animais, apesar de pesados, ainda eram relativamente jovens e possivelmente tiveram certo desenvolvimento dos tecidos ósseo e muscular durante o período experimental. Além disso, a partir dos dados apresentados por Gesualdi Jr. et al. (2006), que avaliaram a composição física dos animais utilizados neste trabalho, observa-se que não há variação expressiva nos valores numéricos nas proporções dos tecidos ósseo, muscular e adiposo com o aumento do peso corporal dos animais.

Os resultados encontrados neste trabalho estão de acordo com os obtidos por Almeida et al. (2001), que constataram elevação das concentrações corporais de $\mathrm{Ca}$, $\mathrm{P} \mathrm{e} \mathrm{K} \mathrm{com} \mathrm{o} \mathrm{aumento} \mathrm{do} \mathrm{peso} \mathrm{corporal} \mathrm{de} \mathrm{mestiços} \mathrm{Holandês-}$ Gir, e por Backes (2003), que verificou o mesmo comportamento de $\mathrm{Na}$ e $\mathrm{K}$ em zebuínos e mestiços leiteiros (F1 Holandês x Gir e F1 Holandês x Guzerá). Martins (2003) verificou elevação das concentrações corporais de $\mathrm{Ca}, \mathrm{P}$, $\mathrm{Na}$ e K por unidade de PCVZ para animais Nelore e de $\mathrm{Mg}$ para mestiços F1 Holandês x Nelore e F1 Caracu x Nelore com o aumento do peso corporal.

Os conteúdos de $\mathrm{Ca}, \mathrm{P}, \mathrm{Mg}$, Na e K por unidade de PCVZ estimados para animais $\mathrm{NeS}$ e $\mathrm{NeC}$ de $400 \mathrm{~kg}$ de PV foram inferiores aos encontrados por Paulino et al. (1999). Martins (2003) obteve maiores concentrações de $\mathrm{Mg}$ e Na e menores de $\mathrm{Ca}, \mathrm{P}$ e K que as observadas neste trabalho. Nos animais $\mathrm{CaS}$, os conteúdos de $\mathrm{Ca}$ por unidade de PCVZ foram

Tabela 4 - Estimativa dos conteúdos de Ca, P, Mg, Na e K por unidade de peso corporal vazio (g/kg de PCVZ), em bovinos Nelore e Caracu1, de acordo com o peso vivo (PV) ou do peso de corpo vazio (PCVZ), kg

Table 4 - Estimates of contents of $\mathrm{Ca}, \mathrm{P}, \mathrm{Mg}, \mathrm{Na}$, and K per unit of empty body weight, of Nellore and Caracu, in function of body weight (BW) or empty body weight (EBW), $\mathrm{kg}$

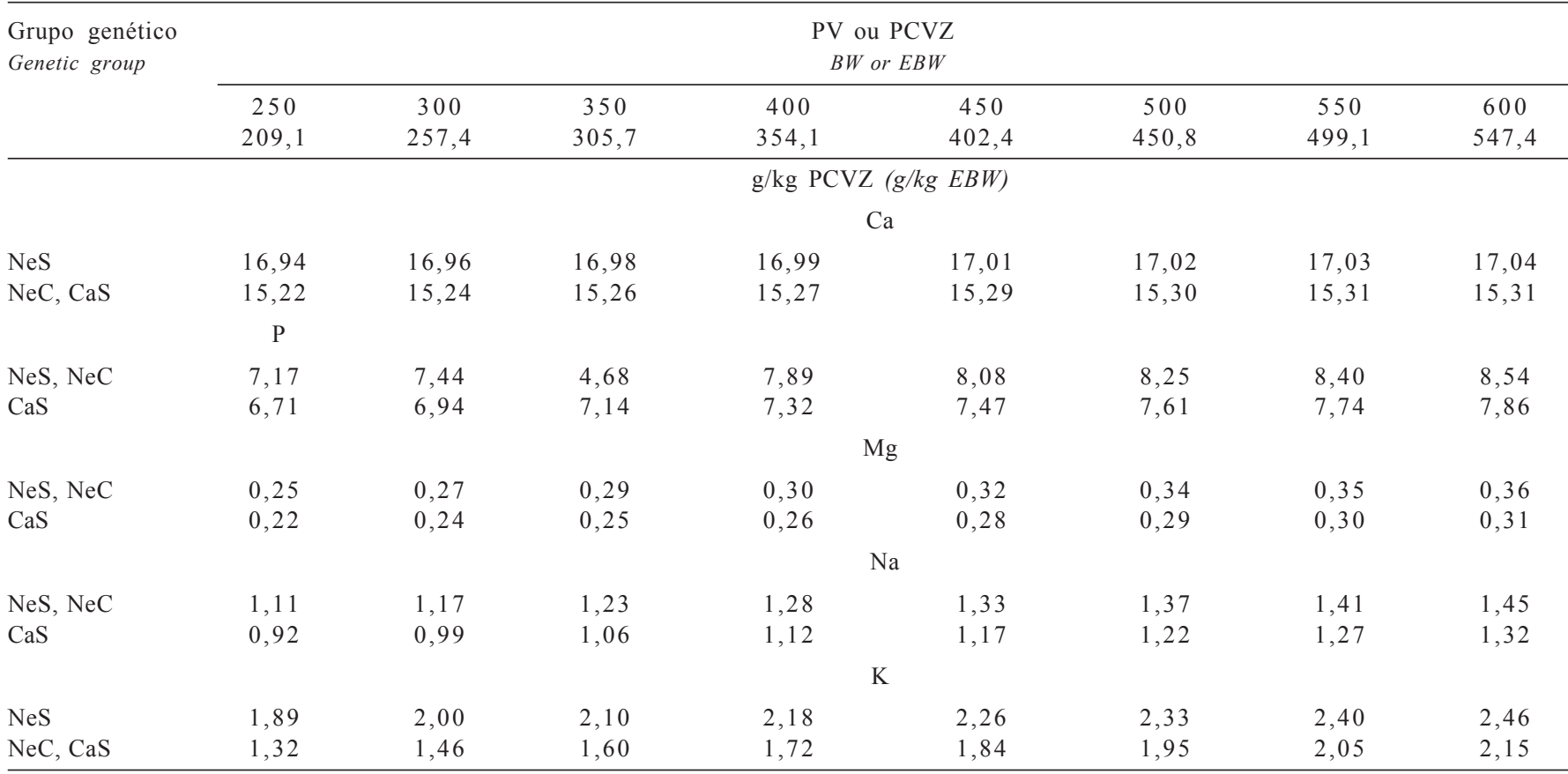

\footnotetext{
${ }^{1} \mathrm{NeS}=$ Nelore selecionado; $\mathrm{NeC}=$ Nelore não-selecionado; CaS = Caracu selecionado.
}

${ }^{1} \mathrm{NeS}=$ Genetically selected Nellore; $\mathrm{NeC}=$ Ordinary Nellore; $\mathrm{CaS}=$ Genetically selected Caracu . 
superiores, enquanto os de $\mathrm{P}, \mathrm{Mg}, \mathrm{Na}$ e $\mathrm{K}$ foram inferiores aos valores médios calculados por Silva et al. (2002b) para animais Holandês. Martins (2003) encontrou concentrações inferiores de $\mathrm{Ca}, \mathrm{P}$ e $\mathrm{K}$ e superiores de $\mathrm{Mg}$ e $\mathrm{Na}$ para mestiços F1 Holandês x Nelore e F1 Caracu x Nelore.

Lana et al. (1992), trabalhando com animais Nelore e mestiços F1 Chianina x Nelore, F1 Holandês x Nelore, F1 Holandês x Gir e 3/4 Holandês x Gir, observaram menores concentrações de $\mathrm{Ca}$, $\mathrm{Na}$ e $\mathrm{K}$ que as estimadas neste experimento para todos os grupos genéticos estudados. Paulino et al. (2004), em estudo com animais anelorados, observaram menores concentrações de $\mathrm{Ca}, \mathrm{P}, \mathrm{Mg}, \mathrm{Na}$ e K que as estimadas neste experimento para $\mathrm{NeS}$ e menores de $\mathrm{Ca}, \mathrm{Pe} \mathrm{Mg}$ que as estimadas neste trabalho para $\mathrm{NeC} \mathrm{e} \mathrm{CaS}$. Porém, estes autores trabalharam com animais castrados, que depositam mais gordura, com maior teor corporal de gordura, e, conseqüentemente, apresentam queda na porcentagem de ossos na carcaça e diluição do conteúdo corporal de minerais.

Derivando-se as equações de regressão anteriormente apresentadas, foram obtidas as equações de predição dos conteúdos de $\mathrm{Ca}, \mathrm{P}, \mathrm{Mg}$, Na e K por kg ganho de PCVZ, os quais, por sua vez, correspondem às exigências líquidas destes macroelementos minerais para ganho de $1 \mathrm{~kg}$ de PCVZ(Tabela 5).
Da mesma forma que para os conteúdos corporais, as exigências líquidas para ganho de peso dos macroelementos minerais estudados também aumentaram com a elevação do peso de corpo vazio dos animais. Esse comportamento também contraria os relatos na literatura de decréscimo nas exigências líquidas para ganho dos macrominerais no corpo vazio com a elevação do PCVZ, como conseqüência do efeito de diluição provocado pelo aumento da gordura corporal, que tem baixo teor de minerais. Além disso, com a elevação do peso e da idade dos animais, ocorre também redução na proporção de ossos na carcaça, o que acarreta queda nas concentrações de $\mathrm{Ca}, \mathrm{P} \mathrm{e} \mathrm{Mg}$, visto que os ossos contêm de 98 a $99 \%$ do $\mathrm{Ca}, 80 \%$ do $\mathrm{P}$ e 65 a $70 \%$ do $\mathrm{Mg}$ corporais.

O comportamento observado neste trabalho difere do relatado por Lana et al. (1992), Coelho da Silva (1995), Fontes (1995), Paulino et al. (1999), Véras et al. (2001), Silva et al. (2002a,b) e Paulino et al. (2004). Todavia, está de acordo com os resultados obtidos por Almeida et al. (2001), que constataram elevação das exigências líquidas de $\mathrm{Ca}, \mathrm{P}$ e K por kg de GPCVZ com o aumento do PCVZ de mestiços Holandês-Gir, e por Backes (2003), que verificou o mesmo comportamento para $\mathrm{Na}$ e $\mathrm{K}$ para zebuínos e mestiços leiteiros. Martins (2003) registrou elevação das exigências líquidas de $\mathrm{Ca}, \mathrm{P}, \mathrm{Na}$ e K por kg de GPCVZ para Nelore e de

Tabela 5 - Exigências líquidas estimadas de $\mathrm{Ca}, \mathrm{P}, \mathrm{Mg}, \mathrm{Na}$ e K, em g por kg de ganho de peso de corpo vazio (g/kg de GPCVZ), de bovinos Nelore e Caracu1, em função do peso vivo (PV) ou do peso de corpo vazio (PCVZ), kg

Table 5 - Estimates of net requirements of $\mathrm{Ca}, \mathrm{P}, \mathrm{Mg}, \mathrm{Na}$, and $\mathrm{K}$ in g per $\mathrm{kg}$ of empty body weight gain ( $\mathrm{g} / \mathrm{kg}$ of EBWG), of Nellore and Caracu ${ }^{1}$, in function of body weight (BW) or empty body weight (EBW), kg

\begin{tabular}{|c|c|c|c|c|c|c|c|c|}
\hline \multirow[t]{3}{*}{$\begin{array}{l}\text { Grupo genético } \\
\text { Genetic group }\end{array}$} & \multicolumn{8}{|c|}{$\begin{array}{c}\text { PV ou PCVZ } \\
B W \text { or } E B W\end{array}$} \\
\hline & 250 & 300 & 350 & 400 & 450 & 500 & 550 & 600 \\
\hline & 209,1 & 257,4 & 305,7 & 354,1 & 402,4 & 450,8 & 499,1 & 547,4 \\
\hline & \multicolumn{8}{|c|}{$\mathrm{g} / \mathrm{kg}$ GPCVZ $(g / k g E B W G)$} \\
\hline & \multicolumn{8}{|c|}{$\mathrm{Ca}$} \\
\hline $\mathrm{NeS}$ & 17,04 & 17,06 & 17,08 & 17,09 & 17,11 & 17,12 & 17,13 & 17,14 \\
\hline \multirow[t]{2}{*}{$\mathrm{NeC}, \mathrm{CaS}$} & 15,32 & 15,34 & 15,35 & 15,37 & 15,38 & 15,39 & 15,40 & 15,41 \\
\hline & \multicolumn{8}{|c|}{$P$} \\
\hline $\mathrm{NeS}, \mathrm{NeC}$ & 8,48 & 8,80 & 9,08 & 9,33 & 9,55 & 9,75 & 9,93 & 10,10 \\
\hline \multirow[t]{2}{*}{$\mathrm{CaS}$} & 7,81 & 8,08 & 8,32 & 8,52 & 8,70 & 8,86 & 9,01 & 9,15 \\
\hline & \multicolumn{8}{|c|}{$\mathrm{Mg}$} \\
\hline $\mathrm{NeS}, \mathrm{NeC}$ & 0,35 & 0,38 & 0,40 & 0,43 & 0,45 & 0,47 & 0,49 & 0,51 \\
\hline \multirow[t]{2}{*}{$\mathrm{CaS}$} & 0,30 & 0,32 & 0,34 & 0,35 & 0,37 & 0,38 & 0,40 & 0,41 \\
\hline & \multicolumn{8}{|c|}{$\mathrm{Na}$} \\
\hline $\mathrm{NeS}, \mathrm{NeC}$ & 1,42 & 1,50 & 1,57 & 1,64 & 1,70 & 1,76 & 1,81 & 1,85 \\
\hline \multirow[t]{2}{*}{$\mathrm{CaS}$} & 1,26 & 1,37 & 1,46 & 1,54 & 1,62 & 1,69 & 1,75 & 1,81 \\
\hline & \multicolumn{8}{|c|}{$\mathrm{K}$} \\
\hline $\mathrm{NeS}$ & 2,41 & 2,55 & 2,67 & 2,78 & 2,88 & 2,98 & 3,06 & 3,14 \\
\hline $\mathrm{NeC}, \mathrm{CaS}$ & 1,99 & 2,21 & 2,41 & 2,60 & 2,77 & 2,94 & 3,10 & 3,25 \\
\hline
\end{tabular}

\footnotetext{
${ }^{1} \mathrm{NeS}=$ Nelore selecionado; $\mathrm{NeC}=$ Nelore não-selecionado; $\mathrm{CaS}=$ Caracu selecionado
}

${ }^{1} \mathrm{NeS}=$ Genetically selected Nellore; $\mathrm{NeC}=$ Ordinary Nellore; $\mathrm{CaS}=$ Genetically selected Caracu . 
Mg para mestiços F1 Holandês x Nelore e F1 Caracu x Nelore com o aumento do peso corporal.

As exigências líquidas para ganho de $1 \mathrm{~kg}$ de $\mathrm{PCVZ}$ estimadas para animais $\mathrm{NeS}$ e $\mathrm{NeC}$ de $400 \mathrm{~kg}$ de $\mathrm{PV}$ foram superiores às encontrados por Paulino et al. (1999), Véras et al. (2001) e Silva et al. (2002a). Para animais CaS, as exigências líquidas de $\mathrm{Ca}, \mathrm{Mg}$, $\mathrm{Na}$ e $\mathrm{K}$ foram superiores e a de $\mathrm{P}$ inferior às estimadas por Silva et al. (2002b) para Holandês. Martins (2003) encontrou exigências inferiores de $\mathrm{Ca}, \mathrm{P}, \mathrm{Na}$ e K e superiores de Mg para mestiços F1 Holandês x Nelore e F1 Caracu x Nelore às obtidas neste estudo.

Lana et al. (1992) e Paulino et al. (2004) observaram exigências líquidas para ganho de peso menores que as estimativas obtidas neste experimento para os três grupos genéticos estudados. Porém, ressalta-se, como discutido anteriormente, que estes autores trabalharam com animais castrados, que depositam gordura mais precocemente, com menor concentração de minerais que os tecidos ósseo e muscular, diluindo o conteúdo corporal de minerais e reduzindo os requisitos líquidos para ganho.

Considerando os valores fixos de exigências recomendados pelo ARC (1980) para Ca, P, Mg, Na e K de, respectivamente, 14,$0 ; 8,0 ; 0,45 ; 1,5$ e 2,0 g/kg de GPCVZ, os valores obtidos neste trabalho para animais de $400 \mathrm{~kg}$ de $\mathrm{PV}$, independentemente do grupo genético, foram superiores para $\mathrm{Ca}, \mathrm{P}, \mathrm{Na}$ e $\mathrm{K}$ e inferiores para $\mathrm{Mg}$. Os valores mais baixos sugeridos por esse Conselho são, em grande parte, explicados pelo fato de terem sido obtidos em animais castrados. Vale salientar que as exigências fixas recomendadas pelo ARC (1980) só seriam válidas se os tecidos ósseo, muscular e adiposo aumentassem na mesma proporção que o peso do animal, fato que, como reconhecido pelo AFRC (1991) e pelo NRC (1996) e demonstrado por vários autores, não ocorre.

Utilizando-se o modelo matemático proposto pelo AFRC (1991) para um bovino de $400 \mathrm{~kg}$ de PV e considerando peso à maturidade de $450 \mathrm{~kg}$ para $\mathrm{NeS}$ e $\mathrm{NeC}$ e de $500 \mathrm{~kg}$ para $\mathrm{CaS}$, os requerimentos líquidos estimados por $\mathrm{kg}$ de ganho seriam de 10,09 e 10,3 g para Ca e de 5,96 e 6,1 g para $\mathrm{P}$, respectivamente. Os valores obtidos neste trabalho foram superiores, tanto para $\mathrm{Ca}$ quanto para $\mathrm{P}$, aos estimados segundo a metodologia proposta pelo AFRC (1991). Coelho da Silva (1995) comparou resultados encontrados no Brasil aos obtidos segundo metodologia preconizada pelo AFRC (1991) e concluiu que as estimativas das exigências líquidas de Ca e P diferiram em até $100 \%$ daquelas propostas pelo AFRC(1991).

O NRC (1996) estima as exigências líquidas de Ca e P para ganho de peso, em função do ganho diário de proteína ou proteína retida em 8,5 e 4,8 g/dia, respectivamente, para animais de $450 \mathrm{~kg}$ de PV ganhando $1 \mathrm{~kg}$ de PV por dia. Os valores encontrados neste estudo foram bem superiores aos estimados por esse conselho.

As exigências dietéticas totais (mantença + ganho de $1 \mathrm{~kg}$ de PV) de Ca, P, Mg, Na e K, em g/dia e em \% da MS total da dieta, são apresentadas, respectivamente, nas Tabelas 6 e 7. As exigências dietéticas totais, quando expressas em $\mathrm{g} /$ dia, aumentaram com o peso vivo dos animais, visto que as exigências de mantença, calculadas em função do PV, estão embutidas dentro das exigências líquidas totais. Comportamento semelhante foi observado por Lana et al. (1992), Paulino et al. (1999), Silva et al. (2002a,b) e Paulino et al. (2004).

Ressalta-se que a ausência de uma metodologia unificada para a estimativa das exigências líquidas para mantença e ganho de peso, bem como, de coeficientes de absorção únicos para cada elemento mineral, constitui uma dificuldade na comparação criteriosa entre os valores de exigências dietéticas estimadas nos diferentes trabalhos sobre o assunto.

As exigências dietéticas totais obtidas neste trabalho para animais $\mathrm{NeS}$ e $\mathrm{NeC}$ com PV de $400 \mathrm{~kg}$ e ganho de $1 \mathrm{~kg}$ de $\mathrm{PV} /$ dia foram maiores para $\mathrm{Ca}, \mathrm{Mg}$ e $\mathrm{Na}$ e menores para $\mathrm{P}$ que as encontradas por Silva et al. (2002a). Martins (2003), no entanto, observou maiores exigências dietéticas de $\mathrm{Ca}, \mathrm{P} \mathrm{e} \mathrm{K}$ e menores de $\mathrm{Mg}$ e $\mathrm{Na}$ que as obtidas neste trabalho. Esses autores utilizaram as perdas endógenas de $\mathrm{Ca}$ e $\mathrm{P}$ segundo o AFRC (1991) e as biodisponibilidades segundo o NRC (1996). Para Mg, Na e K, utilizaram as perdas endógenas e as biodisponibilidades recomendadas pelo ARC (1980).

Para animais $\mathrm{CaS}$ com mesmo peso e taxa de ganho citados, as exigências dietéticas totais encontradas foram maiores para $\mathrm{Ca}, \mathrm{Mg}$, $\mathrm{Na}$ e $\mathrm{K}$ e menores para $\mathrm{P}$ que as obtidas por Silva et al. (2002b) para Holandês. Por outro lado, Martins (2003) observou exigências dietéticas de $\mathrm{Ca}$ e $\mathrm{Na}$ e maiores de P, Mg e K para mestiços F1 Holandês x Nelore e F1 Caracu x Nelore menores que as obtidas neste trabalho.

Lana et al. (1992), utilizando as perdas endógenas e as biodisponibilidades segundo o ARC (1980) para todos os minerais, e Paulino et al. (2004), considerando as perdas endógenas e as biodisponibilidades segundo o NRC (1996), para Ca e P, e segundo o ARC (1980), para os demais minerais, verificaram menores exigências dietéticas totais de minerais que as estimativas obtidas neste experimento para todos os grupos genéticos. Porém, como discutido anteriormente, estes autores trabalharam com animais castrados, cujos respectivos requisitos líquidos de minerais para ganho são menores. 
Tabela 6 - Exigências dietéticas totais (mantença + ganho de 1 kg PV) de Ca, P, Mg, Na e K, em g/dia, de bovinos Nelore e Caracu ${ }^{1}$, em função do peso vivo (PV) ou do peso de corpo vazio (PCVZ), $\mathrm{kg}$

Table 6 - Total dietary requirements (maintenance + gain of $1 \mathrm{~kg} \mathrm{BW}$ ) of $\mathrm{Ca}, \mathrm{P}, \mathrm{Mg}, \mathrm{Na}$, and $\mathrm{K}$, in $\mathrm{g} /$ day, of Nellore and Caracu ${ }^{1}$, in function of body weight (BW) or empty body weight (EBW), kg

\begin{tabular}{|c|c|c|c|c|c|c|c|c|}
\hline \multirow[t]{3}{*}{$\begin{array}{l}\text { Grupo genético } \\
\text { Genetic group }\end{array}$} & \multicolumn{8}{|c|}{$\begin{array}{c}\text { PV ou PCVZ } \\
B W \text { or } E B W\end{array}$} \\
\hline & 250 & 300 & 350 & 400 & 450 & 500 & 550 & 600 \\
\hline & 209,1 & 257,4 & 305,7 & 354,1 & 402,4 & 450,8 & 499,1 & 547,4 \\
\hline & \multicolumn{8}{|c|}{$\mathrm{g} / \mathrm{dia} \quad(\mathrm{g} / \mathrm{day})$} \\
\hline & \multicolumn{8}{|c|}{$\mathrm{Ca}$} \\
\hline $\mathrm{NeS}$ & 40,79 & 42,37 & 43,94 & 45,51 & 47,07 & 48,64 & 50,20 & 51,75 \\
\hline & & & & & & & & \\
\hline $\mathrm{NeS}, \mathrm{NeC}$ & 17,98 & 19,63 & 21,21 & 22,73 & 24,23 & 25,69 & 27,12 & 28,54 \\
\hline $\mathrm{CaS}$ & 17,04 & 18,60 & 20,11 & 21,58 & 23,01 & 24,42 & 25,81 & 27,19 \\
\hline & \multicolumn{8}{|c|}{$\mathrm{Mg}$} \\
\hline $\mathrm{NeS}, \mathrm{NeC}$ & 6,38 & 7,44 & 8,48 & 9,50 & 10,51 & 11,51 & 12,51 & 13,50 \\
\hline $\mathrm{CaS}$ & \multicolumn{8}{|c|}{ K } \\
\hline $\mathrm{NeS}$ & 30,16 & 35,65 & 41,11 & 46,56 & 52,01 & 57,44 & 62,87 & 68,29 \\
\hline $\mathrm{NeC}, \mathrm{CaS}$ & 29,75 & 35,32 & 40,86 & 46,38 & 51,90 & 57,40 & 92,90 & 68,39 \\
\hline
\end{tabular}

${ }^{1} \mathrm{NeS}=$ Nelore selecionado; $\mathrm{NeC}=$ Nelore não-selecionado; CaS = Caracu selecionado.

${ }^{1} \mathrm{NeS}=$ Genetically selected Nellore; $\mathrm{NeC}=$ Ordinary Nellore; CaS = Genetically selected Caracu.

Tabela 7 - Exigências dietéticas totais (mantença + ganho de $1 \mathrm{~kg} \mathrm{PV}$ ) de $\mathrm{Ca}, \mathrm{P}, \mathrm{Mg}, \mathrm{Na}$ e $\mathrm{K}$, em \% da MS total da dieta para consumo de $2,4 \% \mathrm{PV}$, de bovinos Nelore e Caracu ${ }^{1}$, em função do peso vivo (PV) ou do peso de corpo vazio (PCVZ), $\mathrm{kg}$

Table 7 - Total dietary requirements (maintenance + gain of $1 \mathrm{~kg} \mathrm{BW}$ ) of $\mathrm{Ca}, \mathrm{P}, \mathrm{Mg}, \mathrm{Na}$, and $\mathrm{K}$, in \% of total diet DM assuming an intake of $2.4 \% \mathrm{BW}$, of Nellore and Caracu ${ }^{1}$, in function of body weight (BW) or empty body weight (EBW), $\mathrm{kg}$

Grupo genético

Genetic group
PV ou PCVZ

$B W$ or $E B W$

\begin{tabular}{|c|c|c|c|c|c|c|c|}
\hline 250 & 300 & 350 & 400 & 450 & 500 & 550 & 600 \\
\hline 209,1 & 257,4 & 305,7 & 354,1 & 402,4 & 450,8 & 499,1 & 547,4 \\
\hline
\end{tabular}

$\%$ MS total (\% Total DM)

\begin{tabular}{|c|c|c|c|c|c|c|c|c|}
\hline \multirow[b]{2}{*}{$\mathrm{NeS}$} & \\
\hline & 0,68 & 0,59 & 0,52 & 0,47 & 0,44 & 0,41 & 0,38 & 0,36 \\
\hline \multirow[t]{2}{*}{$\mathrm{NeC}, \mathrm{CaS}$} & 0,62 & 0,54 & 0,48 & 0,44 & 0,40 & 0,38 & 0,35 & 0,34 \\
\hline & \multicolumn{8}{|c|}{$\mathrm{P}$} \\
\hline $\mathrm{NeS}, \mathrm{NeC}$ & 0,30 & 0,27 & 0,25 & 0,24 & 0,22 & 0,21 & 0,21 & 0,20 \\
\hline \multirow[t]{2}{*}{$\mathrm{CaS}$} & 0,28 & 0,26 & 0,24 & 0,22 & 0,21 & 0,20 & 0,20 & 0,19 \\
\hline & \multicolumn{8}{|c|}{$\mathrm{Mg}$} \\
\hline $\mathrm{NeS}, \mathrm{NeC}$ & 0,11 & 0,10 & 0,10 & 0,10 & 0,10 & 0,10 & 0,09 & 0,09 \\
\hline \multirow[t]{2}{*}{$\mathrm{CaS}$} & 0,10 & 0,10 & 0,10 & 0,09 & 0,09 & 0,09 & 0,09 & 0,09 \\
\hline & \multicolumn{8}{|c|}{$\mathrm{Na}$} \\
\hline \multirow[t]{2}{*}{$\mathrm{NeS}, \mathrm{NeC}$} & 0,06 & 0,05 & 0,05 & 0,05 & 0,05 & 0,05 & 0,05 & 0,04 \\
\hline & \multicolumn{8}{|c|}{ K } \\
\hline $\mathrm{NeS}$ & 0,50 & 0,50 & 0,49 & 0,49 & 0,48 & 0,48 & 0,48 & 0,47 \\
\hline $\mathrm{NeC}, \mathrm{CaS}$ & 0,50 & 0,49 & 0,49 & 0,48 & 0,48 & 0,48 & 0,48 & 0,47 \\
\hline
\end{tabular}

\footnotetext{
${ }^{1} \mathrm{NeS}=$ Nelore selecionado; $\mathrm{NeC}=$ Nelore não-selecionado; CaS = Caracu selecionado
}

${ }^{1} \mathrm{NeS}=$ Genetically selected Nellore; $\mathrm{NeC}=$ Ordinary Nellore; $\mathrm{CaS}=$ Genetically selected Caracu. 
O AFRC (1991) estimou as exigências dietéticas totais de Ca e P para bovinos de $400 \mathrm{~kg}$ de PV ganhando $1 \mathrm{~kg}$ de $\mathrm{PV} /$ dia em, respectivamente, 28,0 e 25,0 g/dia, considerando uma dieta com metabolizabilidade de 0,60 . As exigências dietéticas encontradas neste trabalho para $\mathrm{Ca}$ foram bem maiores e as de $\mathrm{P}$ menores que as recomendadas por esse conselho. Os valores registrados neste estudo são superiores, tanto para $\mathrm{Ca}$ quanto para $\mathrm{P}$, aos preconizados pelo NRC (1996), de 31,0 e 18,0 g/dia. Ressalta-se que o AFRC (1991) utilizou metodologia diferente da adotada pelo NRC (1996) para o cálculo das exigências líquidas para mantença e ganho de peso, bem como diferentes coeficientes de absorção, os quais foram de 0,68 para Ca e de 0,58 para $P$.

O ARC (1980) estimou as exigências dietéticas totais de $\mathrm{Mg}$, Na e K para bovinos de $400 \mathrm{~kg}$ de $\mathrm{PV}$ ganhando $1 \mathrm{~kg}$ de $\mathrm{PV} /$ dia em, respectivamente, 9,50; 4,64 e 43,38 g/dia. Os valores encontrados neste trabalho para animais $\mathrm{NeS}$ e $\mathrm{NeC}$ são iguais para $\mathrm{Mg}$ e superiores para $\mathrm{Na}$ e K; e, com relação aos animais $\mathrm{CaS}$, as exigências dietéticas totais foram inferiores para $\mathrm{Mg}$ e $\mathrm{Na}$ e superiores para $\mathrm{K}$ às recomendadas pelo ARC (1980). Estas diferenças se devem basicamente às estimativas das exigências líquidas para ganho de peso.

Segundo o NRC (1996), um animal de $400 \mathrm{~kg}$ de PV ganhando $1 \mathrm{~kg}$ de PV/dia e consumindo 2,4\% do PV de MS teria exigências dietéticas totais de $\mathrm{Ca}, \mathrm{P}, \mathrm{Mg} \mathrm{Na}$ e $\mathrm{K}$, em \% da MS total da dieta, de 0,$32 ; 0,19 ; 0,10 ; 0,07$ e $0,60 \%$, respectivamente. Os valores encontrados neste trabalho foram superiores para $\mathrm{Ca}$ e $\mathrm{P}$, praticamente iguais para $\mathrm{Mg}$ e inferiores para $\mathrm{Na}$ e K aos recomendados pelo NRC (1996).

Especificamente para $\mathrm{Mg}$ e $\mathrm{K}$, as exigências líquidas para ganho representam uma pequena parcela das exigências dietéticas totais, tendo em vista a baixa disponibilidade do $\mathrm{Mg}$ alimentar e as elevadas exigências líquidas de $\mathrm{K}$ para mantença, conforme observado e citado por Fontes (1995). Além disso, Coelho da Silva (1995) ressalta que há grandes variações individuais na absorção de $\mathrm{Mg}$ por bovinos, advertindo ainda que essas diferenças podem ocorrer até mesmo em gêmeos homozigóticos da referida espécie.

As diferenças entre os grupos genéticos, quanto às exigências dietéticas totais dos cinco macroelementos minerais estudados, quando expressas em \% da MS total da dieta, foram pouco expressivas, não justificando, portanto, a utilização de tabelas específicas para cada grupo genético.

Os altos valores de exigências dietéticas totais encontrados neste trabalho foram obtidos com animais terminados em confinamento e, portanto, a extrapolação destes valores para a formulação de rações para animais terminados a pasto deve ser vista com reserva.

\section{Conclusões}

Houve efeito do grupo genético sobre o conteúdo corporal dos macroelementos minerais estudados.

As concentrações corporais e as exigências de $\mathrm{Ca}, \mathrm{P}$, $\mathrm{Mg}, \mathrm{Na}$ e $\mathrm{K}$ aumentaram com a elevação do peso corporal dos animais.

As exigências dietéticas totais de $\mathrm{Ca}, \mathrm{P}, \mathrm{Mg}, \mathrm{Na}$ e $\mathrm{K}$, expressas em $\%$ da MS total da dieta, foram semelhantes entre os animais Nelore selecionados, Nelore não-selecionados e Caracu selecionados.

\section{Literatura Citada}

AGRICULTURAL AND FOOD RESEARCH COUNCIL - AFRC. Technical committee on responses to nutrients, Report 6. A reappraisal of the calcium and phosphorous requirements of sheep and cattle. Nutrition Abstract and Reviews, v.61, n.9, p. 576-612, 1991.

AGRICULTURAL RESEARCH COUNCIL - ARC. The nutrient requirements of ruminants livestock. London: Commonwealth Agricultural Bureaux, 1980. 351p.

AlMEIDA, M.I.V.; FONTES, C.A.A.; AlMEIDA, F.Q. et al. Conteúdo corporal e exigências líquidas e dietéticas de macroelementos minerais ( $\mathrm{Ca}, \mathrm{P}, \mathrm{Mg}, \mathrm{Na}$ e $\mathrm{K}$ ) de novilhos mestiços Holandês-Gir em ganho compensatório. Revista Brasileira de Zootecnia, v.28, n.2, p.849-857, 2001.

BACKES, A.A. Composição corporal e exigências de energia, proteína e macroelementos minerais para bovinos mestiços leiteiros e zebu, castrados, em fase de recria e engorda, em confinamento. Viçosa, MG: Universidade Federal de Viçosa, 2003. 97p. Tese (Doutorado em Zootecnia) Universidade Federal de Viçosa, 2003.

COELHO DA SILVA, J.F. Exigências de macroelementos inorgânicos para bovinos: o sistema ARC/AFRC e a experiência no Brasil. In: SIMPÓSIO INTERNACIONAL SOBRE EXIGÊNCIAS NUTRICONAIS DE RUMINANTES, 1., 1995, Viçosa, MG. Anais.. Viçosa, MG: Universidade Federal de Viçosa, 1995. p.467-504.

CONRAD, J.H.; McDOWELL, L.R.; ELLIS, G.L. et al. Minerais para ruminantes em pastejo em regiões tropicais. Tradução de Valéria Pacheco Euclides. Campo Grande: EMBRAPA-CNPGC, 1985. 90p.

FONTES, C.A.A. Composição corporal, exigências líquidas de nutrientes para ganho de peso e desempenho produtivo de animais zebuínos e mestiços europeu-zebu. Resultados experimentais. In: SIMPÓSIO INTERNACIONAL SOBRE EXIGÊNCIAS NUTRICIONAIS DE RUMINANTES, 1., 1995, Viçosa, MG. Anais... Viçosa, MG: Universidade Federal de Viçosa, 1995. p.419-455.

GESUALDI JR., A.; QUEIROZ, A.C.; RESENDE, F.D. et al Características de carcaça de bovinos Nelore e Caracu selecionados para peso aos 378 dias de idade recebendo alimentação restrita e à vontade. Revista Brasileira de Zootecnia, v.35, n.1, p.131-138, 2006.

HANKINS, O.G.; HOWE, P.E. Estimation of the composition of the beef carcasses and cuts. Washington: USDA, 1946. p.1-16 (Technical Bulletin, 926).

LANA, R.P.; FONTES, C.A.A.; PERON, A.J. et al. Composição corporal e do ganho e exigências de energia, proteína e macroelementos minerais ( $\mathrm{Ca}, \mathrm{P}, \mathrm{Mg}, \mathrm{Na}$ e $\mathrm{K}$ ) de novilhos de cinco grupos raciais. 3. Conteúdo corporal e do ganho de peso e exigências de macroelementos minerais. Revista Brasileira de Zootecnia, v.21, n.3, p.538-544, 1992. 
MARTINS, R.G.R. Exigências de energia, proteína e macroelementos minerais ( $\mathrm{Ca}, \mathrm{P}, \mathrm{Na}, \mathrm{K}$ e $\mathrm{Mg}$ ) de bovinos Nelore e mestiços, não castrados, em confinamento. Viçosa, MG: Universidade Federal de Viçosa, 2003. 78p. Tese (Doutorado em Zootecnia) - Universidade Federal de Viçosa, 2003.

NATIONAL RESEARCH COUNCIL - NRC. Nutrients requirements of beef cattle. 7.ed. Washington, D.C.: National Academy Press, 1996. 242p.

PAULINO, M.F.; FONTES, C.A.A.; JORGE, A.M. et al. Composição corporal e exigências de macroelementos minerais $(\mathrm{Ca}, \mathrm{P}, \mathrm{Mg}$, $\mathrm{Na}$ e K) de bovinos não-castrados de quatro raças zebuínas. Revista Brasileira de Zootecnia, v.28, n.3, p.634-641, 1999.

PAULINO, P.V.R.; COSTA, M.A.L.; VALADARES FILHO, S.C. et al. Exigências nutricionais de zebuínos: minerais. Revista Brasileira de Zootecnia, v.33, n.3, p.770-780, 2004.

REGAZZI, A.J. Teste para verificar a identidade de modelos de regressão. Pesquisa Agropecuária Brasileira, v.31, n.1, p. $1-17,1996$.

SILVA, D.J.; QUEIROZ, A.C. Análise de alimentos (métodos químicos e biológicos). 3.ed. Viçosa, MG: Universidade Federal de Viçosa, 2002. 235p.

SILVA, F.F.; VALADARES FILHO, S.C.; ÍTAVO, L.C.V. et al. Composição corporal e requisitos líquidos e dietéticos de macroelementos minerais de bovinos Nelore não-castrados. Revista Brasileira de Zootecnia, v.31, n.2, p.757-764, 2002 a.
SILVA, F.F.; VALADARES FILHO, S.C.; ÍTAVO, L.C.V. et al. Exigências líquidas e dietéticos de energia, proteína e macroelementos minerais de bovinos de corte no Brasil. Revista Brasileira de Zootecnia, v.31, n.2, p.776-792, 2002b.

UNDERWOOD, E.J. The mineral nutrition of livestock. London: Commonwealth Agricultural Bureaux, 1981. 180p.

UNIVERSIDADE FEDERAL DE VIÇOSA - UFV. SAEG - Sistema de análises estatísticas e genéticas. Versão 8.0. Viçosa, MG: 1998. 150p. (Manual do usuário).

VÉRAS, A.S.; VALADARES FILHO, S.C.; COELHO DA SILVA, J.F. et al. Composição corporal e requisitos líquidos e dietéticos de macroelementos minerais de bovinos Nelore não-castrados Revista Brasileira de Zootecnia, v.30, n.3, p.1106-1111, 2001 